\title{
Modelamiento numérico y computacional de la viga de Timoshenko sujeto a cargas puntuales
}

\author{
Frank Henry Acasiete Quispe ${ }^{1}$
}

Resumen: Estudiamos la estabilización uniforme de una clase de sistemas Timoshenko con carga puntual en el extremo libre de la viga. Nuestro resultado principal es demostrar que el semigrupo asociado a este modelo no es exponencialmente estable. Además, demostramos que el semigrupo decae polinomialmente a cero. Cuando el mecanismo de amortiguación es efectivo solo en el límite del ángulo de rotación, la solución también decae polinomialmente con una tasa que depende de los coeficientes del problema. El objetivo de este trabajo es presentar de forma didáctica los resultados contenidos en el artículo [9], usando la teoría de semigrupos vista en [10] y también contribuir con la parte numérica vista en [1].

Palabras clave: Ecuación Diferencial Parcial, viga, semigrupo, estabilidad polinomial.

\section{Numerical and computational modeling of the Timoshenko beam subject to point loads}

\begin{abstract}
We studied the uniform stabilization of a class of Timoshenko systems with tip load at the free end of the beam. Our main result is to prove that the semigroup associated to this model is not exponentially stable. Moreover, we prove that the semigroup decays polynomially to zero. When the damping mechanism is efective only on the boundary of the rotational angle, the solution also decays polynomially with rate depending on the coeficients of the problem. The objective of this work is to present in a didactic way the results obtained in the article [9], using the theory of semigroups used in [10] and also contribute with the numerical part seen in [1]
\end{abstract}

Keywords: Differential partial equations, beam, semigroup, polinomial stability.

Recibido: 15/08/2018. Aceptado: 20/11/2018. Publicado online: 31/12/2018.

\footnotetext{
(C)Los autores. Este artículo es publicado por la Revista PESQUIMAT de la Facultad de Ciencias Matemáticas, Universidad Nacional Mayor de San Marcos. Este es un artículo de acceso abierto, distribuido bajo los términos de la licencia Creative Commons Atribucion-No Comercia-Compartir Igual 4.0 Internacional.(http://creativecommons.org/licenses/by-nc-sa/4.0/) que permite el uso no comercial, distribución y reproducción en cualquier medio, siempre que la obra original sea debidamente citada. Para información, por favor póngase en contacto con revistapesquimat.matematica@unmsm.edu.pe
}

${ }^{1}$ LNCC, Laboratorio Nacional de Computación Científica. e-mail: frankhaq@lncc.br 


\section{Introducción}

Los estudios de las propiedades del sistema de Timoshenko fueron iniciadas en 1921 con el trabajo [16], el cual fue considerando el siguiente problema, siendo estudiado otras propiedades en $[6,13,14]$ y en otros trabajos más.

$$
\begin{aligned}
\rho_{1} \varphi_{t t}-\kappa\left(\varphi_{x}+\psi\right)_{x}=0, & (x, t) \in(0, L) \times(0, T), \\
\rho_{2} \psi_{t t}-b \psi_{x x}+\kappa\left(\varphi_{x}+\psi\right)=0, & (x, t) \in(0, L) \times(0, T),
\end{aligned}
$$

donde $t$ es la variable temporal, $x$ la variable espacial definida en $(0, L)$, siendo $L$ la longitud de la viga.

las funciones $\varphi, \psi$ representan el desplazamiento transversal y el ángulo de rotación de la sección transversal, respectivamente.

Las constantes del sistema son definidas por $\rho_{1}=\rho A, \rho_{2}=\rho I, \kappa=K A G, b=E I$, donde $\rho$ denota la densidad, $A$ el área de la sección transversal, $I$ el área del momento de inercia, $K$ el coeficiente de cizalla $(K<1), E$ y $G$ representan constantes elásticas que dependen del material.

En este trabajo estamos interesados en obtener la estabilización del sistema (1)-(2) donde una extremidad de la viga está presa y la otra está ligada a un cuerpo. Tal cuerpo puede representar un recipiente sellado conteniendo en su interior un material granular.

Este material genera una presión interna, proporcionando así el amortecimiento del movimiento de la viga. La configuración física del problema es mostrada en la Figura 1.

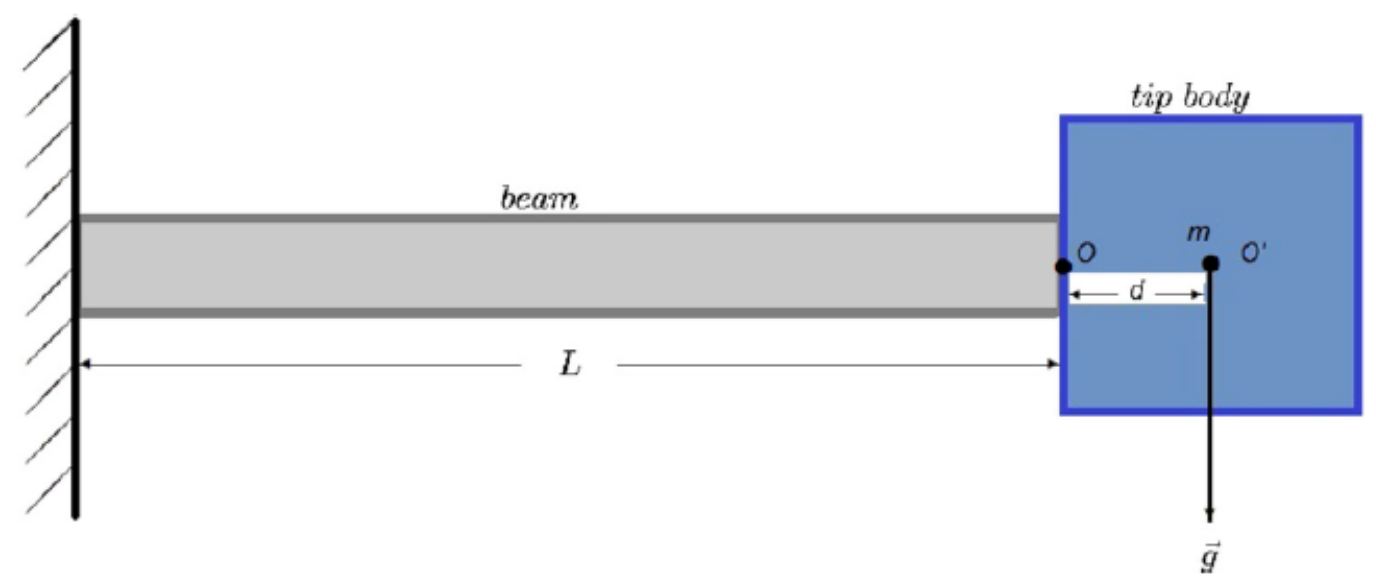

Figura 1: Viga de Timoshenko con cuerpo en la extremidad y parámetros

Tales condiciones sobre las extremidades de la viga serán descritas a seguir. Vamos asumir que la parte presa está localizada a la izquierda, entonces $(\varphi, \psi)$ deben satisfacer en $x=0$

$$
\varphi(0, t)=\psi(0, t)=0 .
$$

Con respecto a la extremidad derecha, vamos asumir que el recipiente se encuentra ligado en $x=L$ con masa $m$, centro de masa $O^{\prime}$ y localizado a una distancia $d$ de la extremidad de la viga. 
Asumimos también que el efecto de amortiguación del material en $\varphi$ y $\psi$ puede ser representado por las constantes $k_{0}$ y $k_{1}$, respectivamente. Con eso tenemos la siguiente condición en $x=L$

$$
m \varphi_{t t}(L, t)+k_{0} \varphi_{t}(L, t)+S(L, t)=0,
$$

donde $S(L, t)=\kappa(L)\left(\varphi_{x}(L, t)+\psi(L, t)\right)$. Los dos primeros terminos denotan la contribución de la inercia del cuerpo en la extremidad. De facto, el componente vertical del término de inercia del recipiente es $m(\varphi+d \sin (\theta))_{t t}$ donde $\theta$ es el ángulo entre el segmento $\overrightarrow{O O^{\prime}}$ y el eje $x$. Mas en la aproximación lineal desconsideramos el término $d \sin (\theta)$. El segundo término representa el amortecimiento proporcionado por el material granular, que es supuesto a ser proporcional al coeficiente de velocidad de amortecimiento $k_{0}$. El término $S(x, t)$ representa la fuerza de cizalla. Similarmente para $\psi$ obtenemos la condición de contorno en la extremidad derecha

$$
I_{m} \psi_{t t}(L, t)+k_{1} \psi_{t}(L, t)+M(L, t)=0,
$$

donde, $M(L, t)=b(L) \psi_{x}(L, t)$ es el momento de flexión y $I_{m}$ es el momento de inercia de la extremidad.

Para ver otros casos de sistema de Timoshenko con disipación, ver [5, 7, 8].

\subsection{Resultados Auxiliares}

Aqui presentaremos algunos resultados de la Teoría de Semigrupos los cuales no demostraremos, el lector interesado puede encontrar la demostración en [12].

Teorema 1.1 (Hille-Yoshida) Un operador lineal $A: D\left(A_{1}\right) \subset X \rightarrow X$ (no limitado), $X$ um espacio de Banach, es un generador de un semimgrupo de clase $C_{0}$ de contracciones $\{S(t)\}$ sí, y solamente sí:

- $A_{1}$ es un operador cerrado y $\overline{D\left(A_{1}\right)}=X$;

- $\quad \mathbb{R}^{+} \subset \rho\left(A_{1}\right)$ y, para todo $\lambda>0$, vale la siguiente desigualdad

$$
\left\|(\lambda I-A)^{-1}\right\| \leq \frac{1}{\lambda}
$$

Teorema 1.2 (Lummer Phillips) Sea $A_{1}: D\left(A_{1}\right) \subset H \rightarrow H$ un operador lineal densamente definido y $H$ un espacio de Hilbert. Entonces:

- Si $A_{1}$ es disipativo y existe $\lambda_{0}>0$, tal que, $\operatorname{Im}\left(\lambda_{0} I-A_{1}\right)=H$. Entonces $A_{1}$ es un generador infinitesimal de un semigrupo de clase $C_{0}$ de contracciones.

- $\quad S i A_{1}$ es el generador infinitesimal de un semigrupo de clase $C_{0}$ de contacciones entonces $\operatorname{Im}\left(\lambda_{0} I-A_{1}\right)=H$, para todo $\lambda>0$ y $A_{1}$ es disipativo.

Del Teorema de Lummer-Phillips sigue un importante resultado que aquí enunciamos como el siguiente corolario.

Corolario 1.3 Sea $A_{1}: D\left(A_{1}\right) \subset H \rightarrow H$ un operador densamente definido, disipativo y tal que $0 \in \rho\left(A_{1}\right)$. Entonces el operador $A_{1}$ es un generador infinitesimal de un semigrupo de clase $C_{0}$ de contracciones.

Con relación al comportamiento asintótico de un semigrupo, tenemos. 
Teorema 1.4 (Borichlev Tomilov [2]) Sea $\{S(t)\}$ un semigrupo de clase $C_{0}$ en un espacio de Hilbert $H$ con generador infinitesimal $A_{1}$, tal que, $i \mathbb{R} \subset \rho\left(A_{1}\right)$. Entonces las siguientes desigualdades son equivalentes

$$
\frac{1}{|\eta|^{\alpha}}\left\|\left(i \eta-A_{1}\right)^{-1}\right\| \leq C, \forall \eta \in \mathbb{R}
$$

$y$

$$
\left\|S(t) A_{1}^{-1}\right\| \leq \frac{c}{t^{\frac{1}{\alpha}}}
$$

\section{Estabilidad polinomial para un sistema de Timoshenko no homogéneo}

Vamos a estudiar el siguiente problema

$$
\begin{aligned}
\rho_{1}(x) \varphi_{t t}-\left(\kappa(x)\left(\varphi_{x}+\psi\right)\right)_{x} & =0 \\
\rho_{2}(x) \psi_{t t}-\left(b(x) \psi_{x}\right)_{x}+\kappa(x)\left(\varphi_{x}+\psi\right) & =0 .
\end{aligned}
$$

Para este sistema vamos a considerar la condición de frontera en $x=0$

$$
\varphi(0, t)=\psi(0, t)=0
$$

y condición de frontera en $x=L$ dada por

$$
\begin{array}{r}
m \varphi_{t t}(L, t)+k_{0} \varphi_{t}(L, t)+\kappa(L)\left(\varphi_{x}(L, t)+\psi(L, t)\right)=0 \\
I_{m} \psi_{t t}(L, t)+k_{1} \psi_{t}(L, t)+b(L) \psi_{x}(L, t)=0 .
\end{array}
$$

y vamos a considerar el sistema sujeto a los siguientes datos iniciales

$$
\varphi(x, 0)=\varphi_{0}(x), \varphi_{t}(x, 0)=\varphi_{1}(x), \psi(x, 0)=\psi_{0}(x), \psi_{t}(x, 0)=\psi_{1}(x) .
$$

\subsection{Formulación abstracta}

Para definir el semigrupo asociado al sistema (8)-(13), vamos a usar la siguiente notación

$$
\varphi_{t}(L, t)=u(t), \psi_{t}(L, t)=v(t)
$$

Entonces de (12)-(13), tenemos que $u$ y $v$ satisfacen el siguiente sistema

$$
\begin{aligned}
m u_{t}(t)+k_{0} u(L, t)+\kappa(L)\left(\varphi_{x}(L, t)+\psi(L, t)\right) & =0, \\
I_{m} v_{t}(t)+k_{1} v(t)+b(L) \psi_{x}(L, t) & =0,
\end{aligned}
$$

con la condición inicial

$$
u(0)=\varphi_{1}(L), v(0)=\psi_{1}(L) .
$$

Vamos a denotar por $U=\left(\varphi, \varphi_{t}, \psi, \psi_{t}, u, v\right)^{\prime}$, donde ' denota el vector transpuesto. Entonces $U$ satisface el siguiente problema de Cauchy

$$
U_{t}=A_{1} U, U(0)=U_{0},
$$


donde $U_{0}=\left(\varphi_{0}, \varphi_{1}, \psi_{0}, \psi_{1}, u_{0}, v_{0}\right)^{\prime}$ y $A_{1}: D\left(A_{1}\right) \subseteq H \rightarrow H$ es dado por

$$
A_{1}=\left(\begin{array}{cccccc}
0 & I & 0 & 0 & 0 & 0 \\
\frac{1}{\rho_{1}}\left[\kappa \partial_{x}\right]_{x} & 0 & \frac{\kappa}{\rho_{1}} \partial_{x} & 0 & 0 & 0 \\
0 & 0 & 0 & I & 0 & 0 \\
-\frac{\kappa}{\rho_{2}} \partial_{x} & 0 & \frac{1}{\rho_{2}}\left[b \partial_{x x}\right]-\frac{\kappa}{\rho_{2}} I & 0 & 0 & 0 \\
-\frac{1}{m} \gamma_{1} & 0 & -\frac{1}{m} \gamma_{2} & 0 & -\frac{k_{0}}{m} I & 0 \\
0 & 0 & -\frac{1}{m} \gamma_{3} & 0 & 0 & -\frac{k_{1}}{m} I
\end{array}\right)
$$

donde $\gamma_{i}, i=1,2,3$, es un operador trazo y es dado por

$$
\gamma_{1}(\varphi)=k(L) \varphi_{x}(L), \gamma_{2}(\psi)=k(L) \psi(L), \gamma_{3}(\psi)=b(L) \psi_{x}(L) .
$$

El espacio de fase $H$ asociado al sistema (18) es dado por

$$
H=H_{*}^{1}(0, L) \times L^{2}(0, L) \times H_{*}^{1}(0, L) \times L^{2}(0, L) \times \mathbb{C}^{2},
$$

donde $H_{*}^{1}(0, L)=\left\{w \in H^{1}(0, L) \mid w(0, L)=0\right\}$. El dominio del operador es dado por

$$
D\left(A_{1}\right)=\left\{U \in H \mid A_{1} U \in H, \Phi(L)=u, \Psi(L)=v\right\}
$$

Vamos a proveer $H$ con el siguiente producto interno

$$
\begin{aligned}
\left(U_{1}, U_{2}\right)_{H}= & \int_{0}^{L} \rho_{1} \Phi_{1} \overline{\Phi_{2}} d x+\int_{0}^{L} \rho_{2} \Psi_{1} \overline{\Psi_{2}} d x+\int_{0}^{L} b \psi_{1, x} \bar{\psi}_{2, x} d x \\
& +\int_{0}^{L} \kappa\left(\varphi_{1, x}+\psi_{1}\right) \overline{\left(\varphi_{2, x}+\psi_{2}\right)} d x+m u_{1}(L) u_{2}(L)+I_{m} v_{1}(L) v_{2}(L),
\end{aligned}
$$

donde $U_{j}=\left(\varphi_{j}, \Phi_{j}, \psi_{j}, \Psi_{j}, u_{j}, v_{j}\right)^{\prime} \in H, j=1,2$. Este produto interno induce una norma (equivalente) en $H$ dada por

$$
\|U\|_{H}^{2}=\int_{0}^{L}\left[\rho_{1}|\Phi|^{2}+\rho_{2}|\Psi|^{2}+b\left|\psi_{x}\right|^{2}+\kappa\left|\varphi_{x}+\psi\right|^{2}\right] d x+m|u(L)|^{2}+I_{m}|v(L)|^{2} .
$$

\subsection{Propiedades del operador $A_{1}$}

Vamos a enunciar algunas propiedades que el operador $A_{1}$ satisface. Tales propiedades implicarán en la buena formulación del problema.

Lema 2.1 El operador $A_{1}: D\left(A_{1}\right) \subset H \rightarrow H$, es disipativo.

Demostración: Sea $U=(\varphi, \Phi, \psi, \Psi, u, v)^{\prime} \in D\left(A_{1}\right)$. Entonces

$$
\begin{aligned}
& A_{1} U=\left(\Phi, 1 / \rho_{1}\left[\kappa \varphi_{x}\right]_{x}+\kappa / \rho_{1} \psi_{x}, \Psi,-\kappa / \rho_{2} \varphi_{x}-1 / \rho_{2}\left[\kappa \psi_{x}\right]_{x}-\kappa / \rho_{2} \psi,\right. \\
&\left.1 / m \gamma_{1} \varphi-1 / m \gamma_{2} \psi-k_{0} / m u,-1 / I_{m} \gamma_{3} \psi-k_{1} / I_{m} v\right) .
\end{aligned}
$$

Aplicando integración por partes, obtenemos

$$
\operatorname{Re}\left(A_{1} U, U\right)_{H}=-k_{0}|u|^{2}-k_{1}|v|^{2} \leq 0 .
$$

Antes de demostrar la próxima propiedad vamos a considerar la ecuación resolvente

$$
i \lambda U-A_{1} U=F,
$$


donde $U=(\varphi, \Phi, \psi, \Psi, u, v)^{\prime} \in H$ y $F=\left(f_{1}, f_{2}, f_{3}, f_{4}, f_{5}, f_{6}\right)^{\prime} \in H$ y $t \in R$. En términos de las componentes la ecuación resolvente es escrita como

$$
\begin{aligned}
\lambda \varphi-\Phi & =f_{1} \\
\lambda \rho_{1} \Phi-\left[\kappa\left(\varphi_{x}+\psi\right)\right]_{x} & =f_{2} \\
\lambda \psi-\Psi & =f_{3}, \\
\lambda \rho_{2} \Psi-\left[b \psi_{x}\right]_{x}+\kappa\left(\varphi_{x}+\psi\right) & =f_{4}, \\
m \lambda u+k_{0} u+\kappa(L)\left(\varphi_{x}+\psi\right)(L) & =f_{5}, \\
I_{m} \lambda v+k_{1} v+b(L) \psi_{x}(L) & =f_{6} .
\end{aligned}
$$

Tomando el producto interno de la ecuación resolvente con $U$ y usando el resultado del Lema anterior tenemos

$$
k_{0}|u|^{2}+k_{1}|v|^{2}=\operatorname{Re}(F, U) .
$$

Lema $2.20 \in \rho\left(A_{1}\right)$.

Demostración: Tomando $\lambda=0$ en (23)-(28) obtenemos

$$
\begin{array}{r}
-\left[\kappa\left(\varphi_{x}+\psi\right)\right]_{x}=f_{2} \in L^{2}(0, L) \\
-\left[b \psi_{x}\right]_{x}+\kappa\left(\varphi_{x}+\psi\right)=f_{4} \in L^{2}(0, L) \\
\kappa(L)\left(\varphi_{x}(L)+\psi(L)\right)=-k_{0} f_{1}(L)+f_{5} \in C \\
b(L) \psi_{x}(L)=-k_{1} f_{3}(L)+f_{6} \in C \\
\varphi(0, t)=\psi(0, t)=0 .
\end{array}
$$

Para garantizar la solución de este problema, vamos a considerar la siguiente forma bilineal definida en $\left[H_{*}^{1}(0, L) \times H_{*}^{1}(0, L)\right]^{2}$

$$
a\left(\left(\varphi^{1}, \psi^{1}\right),\left(\varphi^{2}, \psi^{2}\right)\right)=\int_{0}^{L} \kappa\left(\varphi_{x}^{1}+\psi^{1}\right) \overline{\left(\varphi_{x}^{2}+\psi^{2}\right)}+b \psi_{x}^{1} \overline{\psi_{x}^{2}} d x+\alpha \varphi^{1}(L) \varphi^{2}(L) .
$$

y la forma lineal

$$
F(\varphi, \psi)=\int_{0}^{L} f_{2} \varphi d x+\int_{0}^{L} f_{4} \psi d x+\varphi(L)\left(-k_{0} f_{1}(L)+f_{5}\right)+\psi\left(-k_{1} f_{3}(L)+f_{6}\right)
$$

Con respecto a la forma $a(\cdot, \cdot)$ tenemos las siguientes propiedades:

- $a(\cdot, \cdot)$ es bilineal;

- $a(\cdot, \cdot)$ es continua. De facto,

$$
\begin{aligned}
& \left|a\left(\varphi^{1}, \psi^{1}, \varphi^{2}, \psi^{2}\right)\right| \\
& \quad \leq \int_{0}^{L}\left[\kappa\left|\varphi_{x}^{1}\right|\left|\varphi_{x}^{2}\right|+b\left|\psi_{x}^{1}\right|\left|\psi_{x}^{2}\right|+\kappa\left|\psi_{x}^{1}\right|\left|\varphi^{1}\right|+\kappa\left|\varphi^{2}\right|\left|\psi_{x}^{2}\right|+\left|\psi^{1}\right|\left|\psi^{2}\right|\right] d x \\
& \quad+\kappa\left|(L)\left\|\varphi^{1}(L)\right\| \varphi^{2}(L)\right| \\
& \quad \leq \kappa\left\|\varphi_{x}^{1}\right\|\left\|\varphi_{x}^{2}\right\|+b\left\|\psi_{x}^{1}\right\|\left\|\psi_{x}^{2}\right\|+\kappa\left\|\psi_{x}^{1}\right\|\left\|\varphi^{1}\right\|+\kappa\left\|\varphi^{2}\right\|\left\|\psi_{x}^{2}\right\|+\left\|\psi^{1}\right\|\left\|\psi^{2}\right\| .
\end{aligned}
$$

y esto implica que

$$
\left|a\left(\varphi^{1}, \varphi^{2}, \psi^{1}, \psi^{2}\right)\right| \leq C\left\|\left(\varphi^{1}, \psi^{1}, \varphi^{2}, \psi^{2}\right)\right\|_{\left[H_{*}^{1}(0, L) \times H_{*}^{1}(0, L)\right]^{2}} .
$$

- $\quad a(\cdot, \cdot)$ es coerciva. En efecto, para verificar la coercividad, vamos a considerar la identidad 


$$
-\kappa \varphi_{x x}=\kappa \psi_{x}-\kappa\left(\varphi_{x x}+\psi_{x}\right)
$$

y multiplicando por $\bar{\varphi}$ obtenemos

$$
\alpha \varphi(L)^{2}+\kappa \bar{\varphi}(L) \psi(L)+\kappa \int_{0}^{L}\left|\varphi_{x}\right|^{2} d x=-\kappa \int_{0}^{L} \psi_{x} \bar{\varphi} d x+\kappa \int_{0}^{L}\left(\varphi_{x}+\psi\right) \bar{\varphi}_{x} d x+\alpha \varphi(L)^{2} .
$$

Usando la desigualdad de Poincaré dada como el Corolario 2.6.2 de [1], existe una constante $C>0$ tal que

$$
\int_{0}^{L}\left|\varphi_{x}\right|^{2} d x \leq c \int_{0}^{L}\left|\psi_{x}\right|^{2} d x+C \int_{0}^{L}\left(\varphi_{x}+\psi\right)^{2} d x+C_{\epsilon}|\varphi(L)|^{2}+\epsilon|\psi(L)|^{2} .
$$

Por otro lado, note que

$$
\begin{aligned}
\int_{0}^{L}|\psi|^{2} d x & =\int_{0}^{L}\left(\varphi_{x}+\psi\right)^{2} d x-\int_{0}^{L}\left|\varphi_{x}\right|^{2} d x-2 \operatorname{Re} \int_{0}^{L} \varphi_{x} \bar{\psi} d x \\
& \leq \int_{0}^{L}\left(\varphi_{x}+\psi\right)^{2} d x+c \int_{0}^{L}\left|\varphi_{x}\right|^{2} d x+\frac{1}{2} \int_{0}^{L}|\psi|^{2} d x .
\end{aligned}
$$

De esto sigue que

$$
\int_{0}^{L}|\psi|^{2} d x \leq C \int_{0}^{L}\left(\varphi_{x}+\psi\right)^{2} d x+C \int_{0}^{L}\left|\varphi_{x}\right|^{2} d x .
$$

Usando (35) y la Desigualdad de Poincaré, obtenemos

$$
\int_{0}^{L}|\psi|^{2}+|\varphi|^{2} d x \leq C \int_{0}^{L}\left(\varphi_{x}+\psi\right)^{2} d x+C \int_{0}^{L}|\psi|^{2} d x+C_{\epsilon}|\varphi(L)|^{2}+\epsilon|\psi(L)|^{2} .
$$

Desde

$$
|\psi(L)|^{2} \leq C \int_{0}^{L}|\psi|^{2}+\left|\psi_{x}\right|^{2} d x
$$

para $\epsilon$ suficientemente pequeño, encontramos

$$
\int_{0}^{L}|\psi|^{2}+|\varphi|^{2} d x \leq C \int_{0}^{L}\left(\varphi_{x}+\psi\right)^{2} d x+C \int_{0}^{L}|\psi|^{2} d x+c_{\epsilon}|\varphi(L)|^{2} .
$$

Con respecto a la forma $F(\cdot)$ tenemos las siguientes propiedades:

- $\quad F$ es lineal;

- $F$ es continua.

Por el Lema de Lax-Milgran, se tiene la buena formulación del problema y en particular $0 \in$ $\rho\left(A_{1}\right)$.

Lema 2.3 El dominio del operador $A_{1}$ es denso en $H$, esto es $\overline{D\left(A_{1}\right)}=H$

Demostración: Usaremos la prueba dada en el Lema 3.2.3 dada en [3]. Como $D\left(A_{1}\right) \subset H$, sabemos que

$$
\overline{D\left(A_{1}\right)} \oplus D\left(A_{1}\right)^{\perp}=H .
$$

Así, para concluir el resultado es suficiente probar que

$$
D\left(A_{1}\right)^{\perp}=\{0\} .
$$


donde 0 representa el elemento nulo en el espacio $H$. Para esto, sea $F \in H$, tal que

$$
(W, F)_{H}=0, \forall W \in D\left(A_{1}\right) .
$$

Sabemos que, para todo $\lambda>0$ existe un único $U \in D\left(A_{1}\right)$, donde

$$
\lambda U-A_{1} U=F .
$$

Entonces,

$$
\left(W, \lambda U-A_{1} U\right)_{H}=0, \forall W \in D\left(A_{1}\right) .
$$

Luego, para $W=U$, sigue

$$
\lambda\|U\|_{H}^{2}-\left(U, A_{1} U\right)_{H}=0
$$

Tomando la parte real y usando el hecho de $A_{1}$ sea un operador disipativo, obtenemos $U=0$, lo que implica $F=0$.

De los Lemas y del Corolario 1.3 sigue que $A_{1}$ genera un semigrupo de clase $C_{0}$ de contracciones. Así sigue la buena colocación del problema de Timoshenko (8)-(13).

\section{Tasa de decaimento polinomial}

El principal objetivo es enunciar y demostrar el resultado de estabilidad polinomial. La demostración es basada en el reciente trabajo de Borichev-Tomilov [2]. El resultado es escrito en el siguiente Teorema.

Teorema 3.1 La solución fuerte del sistema de Timoshenko (8)-(13) con carga puntual decae polinomialmente, esto es, existe una constante postiva $C$, tal que

$$
\|U(t)\| \leq \frac{C}{t^{\frac{1}{2}}}\left\|U_{0}\right\|_{D\left(A_{1}\right)} .
$$

la constante $C$ viene como consecuencia del Teorema 1.4.

Antes de probar el Teorema 3.1 vamos primero a introducir algunas notaciones y también a probar un resultado auxiliar.

$$
\begin{gathered}
I_{\varphi}(\beta)=\rho_{1} q_{1} \kappa|\Phi(\beta)|^{2}+q_{1}\left|\kappa\left(\varphi_{x}(\beta)+\psi(\beta)\right)\right|^{2}, \\
I_{\psi}(\beta)=\rho_{2} q_{2} b|\Psi(\beta)|^{2}+q_{2}\left|b \psi_{x}(\beta)\right|^{2},
\end{gathered}
$$

donde $q_{1}$ y $q_{2}$ son funciones continuas definidas en el próximo Lema.

$$
N^{2}=\int_{0}^{L}\left[\rho_{1}|\Phi|^{2}+\rho_{2}|\Psi|^{2}+b\left|\psi_{x}\right|^{2}+\kappa\left|\varphi_{x}+\psi\right|^{2}\right] d x .
$$

Lema 3.2 Suponemos que $(\varphi, \Phi, \psi, \Psi, u, v)$ sea una solución fuerte para el sistema (23)- (28). Entonces existe una constante positiva $C$ tal que

$$
N^{2} \leq C\left[I_{\varphi}(\beta)+I_{\psi}(\beta)+\|F\|_{H}^{2}\right]
$$

$y$

$$
I_{\varphi}(\beta)+I_{\psi}(\beta) \leq C\left[N^{2}+\|F\|_{H}^{2}\right],
$$

para $\beta=0, L$. 
Demostración: Vamos primero a probar las desigualdades para el caso $\beta=L$. Sea $\lambda \in i \mathbb{R}$ y $q_{1} \in C^{2}(0, L)$ tal que $q_{1}(0)=0$. Multiplicando la ecuación (24) por $q_{1} \bar{S}$, donde $S=\kappa\left(\varphi_{x}+\psi\right)$, obtenemos

$$
\int_{0}^{L} \lambda \rho_{1} \Phi q_{1} \bar{S} d s-\int_{0}^{L} S_{x} q_{1} \bar{S} d s=\int_{0}^{L} f_{2} q_{1} \bar{S} d s
$$

Usando (25) y (26), el primer término puede ser escrito como

$$
\begin{aligned}
\int_{0}^{L} \lambda \rho_{1} \Phi q_{1} \bar{S} d x & =\int_{0}^{L} \lambda \rho_{1} \Phi q_{1} \kappa \overline{\left(\varphi_{x}+\psi\right)} d x \\
& =-\int_{0}^{L} \rho_{1} \Phi q_{1} \kappa \overline{\lambda \varphi_{x}}-\int_{0}^{L} \rho_{1} \Phi q_{1} \kappa \overline{\lambda \psi} d x \\
& =-\int_{0}^{L} \rho_{1} \Phi q_{1} \kappa \overline{\Phi_{x}}-\int_{0}^{L} \rho_{1} q_{1} \kappa \Phi \bar{\Psi} d x-\int_{0}^{L} \rho_{1} q_{1} \kappa \Phi \overline{\left[\left[f_{3}\right]_{x}+f_{4}\right]} d x
\end{aligned}
$$

Tomando la parte real obtenemos

$$
\operatorname{Re}\left\{\int_{0}^{L} \lambda \rho_{1} \Phi q_{1} \bar{S} d s\right\}=-\frac{1}{2} \int_{0}^{L} \rho_{1} q_{1} \kappa \frac{d}{d x}|\Phi|^{2} d s-\operatorname{Re}\left\{\int_{0}^{L} \rho_{1} q_{1} \kappa \Phi \bar{\Psi} d s\right\}+R_{1},
$$

donde $R_{1}$ contiene términos con $f_{3}$ y $f_{4}$ y satisface

$$
\left|R_{1}\right| \leq C N\|F\|_{H}
$$

Note que

$$
\begin{aligned}
\frac{1}{2} \int_{0}^{L} \rho_{1} q_{1} \kappa \frac{d}{d x}|\Phi|^{2} d x & =\left.\frac{1}{2} \rho_{1} q_{1} \kappa|\Phi|^{2}\right|_{0} ^{L}-\frac{1}{2} \int_{0}^{L}\left[\rho_{1} q_{1} \kappa\right]_{x}|\Phi|^{2} d x \\
& =\frac{1}{2}\left(\rho_{1} q_{1} \kappa|\Phi|^{2}\right)(L)-\frac{1}{2} \int_{0}^{L}\left[\rho_{1} q_{1} \kappa\right]_{x}|\Phi|^{2} d x .
\end{aligned}
$$

De donde sigue que

$$
\operatorname{Re}\left\{\int_{0}^{L} \lambda \rho_{1} \Phi q_{1} \bar{S} d s\right\}=-\frac{1}{2}\left(\rho_{1} q_{1} \kappa|\Phi|^{2}\right)(L)+\frac{1}{2} \int_{0}^{L}\left[\rho_{1} q_{1} \kappa\right]_{x}|\Phi|^{2} d x-\operatorname{Re}\left\{\int_{0}^{L} \rho_{1} q_{1} \kappa \Phi \bar{\Psi} d s\right\}+R_{1} .
$$

Vamos a analizar la segunda integral

$$
\int_{0}^{L} S_{x} q_{1} \bar{S}=\frac{1}{2} \int_{0}^{L} q_{1} \frac{d}{d x}|S|^{2} d x
$$

Combinando estas identidades con (3.2), obtenemos

$$
\frac{1}{2} I_{\varphi}(t)-\frac{1}{2} \int_{0}^{L}\left(\left[\rho_{1} q_{1} \kappa\right]_{x}|\Phi|^{2}+q_{1, x}\left|\kappa\left(\varphi_{x}+\psi\right)\right|^{2}\right) d x-\underbrace{\int_{0}^{L} \rho_{1} q_{1} \kappa \Phi \bar{\Psi} d x}_{J_{1}}=R_{2}
$$

donde $R_{2}=-R_{1}+\int_{0}^{L} f_{2} q_{1} \bar{S} d x$ y satisface la siguiente estimativa

$$
\left|R_{2}\right| \leq c N\|F\|
$$


Tomando ahora $q_{2} \in C^{2}(0, L)$ tal que $q_{2}(0)=0$, y multiplicando la segunda ecuación en $(24)$ por $q_{2} b \overline{\psi_{x}}$ obtenemos

$$
\int_{0}^{L} \lambda \rho_{2} \Psi q_{2} b \overline{\psi_{x}} d x-\int_{0}^{L}\left[b \psi_{x}\right]_{x} q_{2} b \overline{\psi_{x}} d x+\int_{0}^{L} \kappa\left(\varphi_{x}+\psi\right) q_{2} b \overline{\psi_{x}} d x=\int_{0}^{L} f_{4} q_{2} b \overline{\psi_{x}} d x
$$

Vamos a usar la siguiente notación

$$
\begin{aligned}
& J_{2}=\int_{0}^{L} \lambda \rho_{2} \Psi q_{2} b \overline{\psi_{x}} d x \\
& J_{3}=\int_{0}^{L}\left[b \psi_{x}\right]_{x} q_{2} b \overline{\psi_{x}} d x
\end{aligned}
$$

$\mathrm{y}$

$$
J_{4}=\int_{0}^{L} \kappa\left(\varphi_{x}+\psi\right) q_{2} b \overline{\psi_{x}} d x
$$

Estimaremos primero la integral $J_{1}$. Usando las ecuaciones $(25)$ y (26) podemos escribir $J_{2}$ como

$$
\begin{aligned}
J_{2} & =-\int_{0}^{L} \rho_{2} \Psi q_{2} b \overline{\left(\Psi_{x}+\left[f_{3}\right]_{x}\right)} d x \\
& =-\frac{1}{2} \int_{0}^{L} \rho_{2} q_{2} b \frac{d}{d x}|\Psi|^{2} d x-\int_{0}^{L} \rho_{2} \Psi q_{2} b \overline{\left[f_{3}\right]_{x}} d x \\
& =-\frac{1}{2} \rho_{2} q_{2} b|\Psi|^{2}+\frac{1}{2} \int_{0}^{L}\left[\rho_{2} q_{2}\right]_{x}|\Psi|^{2} d x+\int_{0}^{L} \rho_{2} \Psi q_{2} b \overline{\left[f_{3}\right]_{x}} d x
\end{aligned}
$$

Integrando por partes resulta que $J_{3}$

$$
\begin{aligned}
J_{3} & =\int_{0}^{L}\left[b \psi_{x}\right]_{x} q_{2} b \overline{\psi_{x}} d x \\
& =\int_{0}^{L}\left[b \psi_{x}\right]_{x} q_{2} b \overline{\left(\Psi_{x}+\left[f_{3}\right]_{x}\right)} d x \\
& =\int_{0}^{L}\left[b\left(\Psi_{x}+\left[f_{3}\right]_{x}\right)\right]_{x} b \overline{\left(\Psi_{x}+\left(\left[f_{3}\right]_{x}\right)\right.} d x \\
& =\frac{1}{2} \int_{0}^{L} b q_{2} \frac{d}{d x}\left|\Psi_{x}+\left[f_{3}\right]_{x}\right|^{2} d x \\
& =\frac{1}{2} b q_{2}\left|\Psi_{x}+\left[f_{3}\right]_{x}\right|^{2}||_{0}^{L}-\frac{1}{2} \int_{0}^{L}\left[q_{2} b\right]_{x}\left|\Psi_{x}+\left[f_{3}\right]_{x}\right|^{2} d x \\
& =\frac{1}{2} b q_{2}\left|\Psi_{x}+\left[f_{3}\right]_{x}\right|^{2}(L)-\frac{1}{2} \int_{0}^{L}\left[q_{2} b\right]_{x}\left|\Psi_{x}+\left[f_{3}\right]_{x}\right|^{2} d x
\end{aligned}
$$

Usando (23) y (24), obtenemos

$$
\begin{aligned}
J_{4} & =-\int_{0}^{L}\left[\kappa\left(\varphi_{x}+\psi\right)\right]_{x} q_{2} b \bar{\psi} d x-\int_{0}^{L} \kappa\left(\varphi_{x}+\psi\right)\left[q_{2} b\right]_{x} \bar{\psi} d x+P(L) \\
& =\int_{0}^{L} \rho_{1} \Phi q_{2} \overline{b\left(\Psi+f_{3}\right)} d x+\frac{1}{\lambda} \int_{0}^{L} \kappa\left(\varphi_{x}+\psi\right)\left[q_{2} b\right]_{x} \overline{\left(\Psi+f_{3}\right)} d x+R_{3}+P(L) \\
& =\int_{0}^{L} \rho_{2} q_{2} b \Phi \bar{\Psi} d x+P(L)+R_{4},
\end{aligned}
$$


donde $P(L)=q_{2}(L) b \psi(L) k(L)\left(\varphi_{x}+\psi\right)(L)$ y $R_{4}$ tal que

$$
\left|R_{4}\right| \leq C N\|F\|+\frac{c}{|\lambda|} N^{2}
$$

Sumando $J_{2}, J_{3}$ e $J_{4}$

$$
\frac{1}{2} I_{\psi}(L)-\int_{0}^{L}\left(\left[\rho_{2} q_{2} b\right]_{x}|\Psi|^{2}+q_{2, x}\left|b \psi_{x}\right|^{2}\right) d x+\underbrace{\int_{0}^{L} \rho_{2} q_{2} b \Phi \bar{\Psi} d x}_{J_{5}}=\operatorname{Re}\left\{\left(P(L)+R_{4}\right)\right\}
$$

Entonces, escogemos $q_{1}$ y $q_{2}$ tal que

$$
\rho_{2} q_{2} b=\int_{0}^{x} e^{n s} d s, \quad \rho_{1} q_{1} \kappa=\int_{0}^{x} e^{n s} d s .
$$

Debido a esto las integrales $J_{1}$ y $J_{5}$ son iguales. Sumando las igualdades (42) y (44), concluimos que

$$
\int_{0}^{L}\left[\rho_{1} q_{1} \kappa\right]_{x}|\Phi|^{2}+q_{1, x}\left|\kappa\left(\varphi_{x}+\psi\right)\right|^{2}+\left[\rho_{2} q_{2} b\right]_{x}|\Psi|^{2}+q_{2, x}\left|b \psi_{x}\right|^{2} d s=I_{\psi}(L)+I_{\phi}(L)+R_{5},
$$

donde $R_{5}$ es tal que

$$
\left|R_{5}\right| \leq C N\|F\|+\frac{c}{|\lambda|} N^{2}
$$

Denotamos por $q_{3}:=\rho_{2} q_{2} b=\frac{\rho_{2}}{\rho_{1}} \int_{0}^{x} e^{n s} d s$. Por tanto, debemos estimar la derivada de $q_{i}(x)=$ $\gamma_{i}(x) \int_{0}^{x} e^{n s} d x$, con $i=1,2$ donde $\gamma$ satisface $\gamma_{0} \leq \gamma(x) \leq \gamma_{1}$ para $0<\gamma_{0}<\gamma_{1}$. Para $n$ suficientemente grande, obtenemos

$$
\begin{aligned}
q_{x} & =e^{n x} \gamma+\gamma^{\prime}(x) \int_{0}^{x} e^{n s} d s \\
& =\frac{n e^{n x} \gamma+\gamma^{\prime}\left[e^{n x}-1\right]}{n} \\
& \geq \frac{1}{n}\left(\left[n \gamma_{0}-\gamma^{\prime}\right] e^{n x}+\gamma^{\prime}\right) \\
& \geq \frac{1}{n}\left(\frac{3}{4} n \gamma_{0} e^{n x}-2\left\|\gamma^{\prime}\right\|_{\infty}\right) \\
& \geq \frac{\gamma_{0}}{2} e^{x n} .
\end{aligned}
$$

Esto implica que existe una constante $c_{2}>0$ tal que

$$
\int_{0}^{L}\left[\left[\rho_{1} q_{1} \kappa\right]_{x}|\Phi|^{2}+q_{1, x}\left|\kappa\left(\varphi_{x}+\psi\right)\right|^{2}+\left[\rho_{2} q_{2} b\right]_{x}|\Psi|^{2}+q_{2, x}\left|b \psi_{x}\right|^{2}\right] d s \geq \frac{c_{2}}{n} N^{2} .
$$

Por la definición de $N^{2}$

$$
N^{2} \leq C_{0}\left[I_{\psi}+I_{\phi}+N\|F\|+\frac{1}{|\lambda|} N^{2}\right]
$$

donde $C_{0}$ no depende de $n$. También tenemos

$$
I_{\psi}+I_{\varphi} \leq C_{0}\left[N^{2}+N\|F\|_{H}\right] .
$$

Tomando $\lambda$ suficientemente grande, nuestra conclusión es comprobada.

\section{Demostración del Teorema 3.1:}


Paso 1: $i \mathbb{R} \subset \rho\left(A_{1}\right)$. De facto, una vez que $A_{1}$ es un operador cerrado y $D\left(A_{1}\right)$ tiene imersión compacta en el espacio $H$ concluimos que el espectro $\sigma\left(A_{1}\right)$ es discreto. Así, para concluir es necesario mostrar que no hay autovalores imaginarios. Por definición, para ciertos valores el operador $\left(A_{1}-\lambda I\right)$ no es inyectivo y por tanto no puede definirse una inversa. Esos valores conforman el espectro discreto. Obviamente la ecuación 45 sólo tiene soluciones para valores del espectro discreto, y una de esas soluciones se llama vector propio. Por contradicción, vamos suponer que existe un valor propio imaginario, usamos la misma definición dada en el Teorema 3.3 de [9]. Sea $i \lambda \in \sigma\left(A_{1}\right)$, entonces existe $U \neq 0$ satisfaciendo

$$
A_{1} U=i \lambda U
$$

Tomando produto interno en $H$ de esta identidad con $U$ y usando $(4,11)$, obtenemos que

$$
u=v=0, \quad \Psi(L)=\Phi(L)=0 .
$$

Esto implica que

$$
\psi(L)=\Phi(L)=\Phi_{x}(L)=\psi_{x}(L)=0 .
$$

Considerando el sistema (23)-(28) con $F=0$ como un problema de valor inicial con los datos nulos. Por tanto $\varphi=\psi=0$, esto implica que $U=0$. Mas eso es una contradicción y así tenemos que $i \mathbb{R} \subset \rho\left(A_{1}\right)$.

Paso 2: El operador $A_{1}$ satiface la primera estimativa, que es la ecuación (6), del Teorema 1.4, dada en [2] con $\alpha=2$. En efecto, usando el Lema 3.2 para la primera desigualdad, para la segunda desigualdad usamos (22) y, concluímos, para $|\lambda|$ grande, que

$$
\begin{aligned}
\|U\|^{2} & \leq C\left[I_{\varphi}+I_{\psi}+\|F\|_{H}^{2}\right] \\
& \leq C\left[|\lambda|^{2}|u|^{2}+|\lambda|^{2}|v|^{2}+\|F\|_{H}^{2}\right] \\
& \leq C|\lambda|^{2}\|U\|_{H}\|F\|_{H}+C\|F\|_{H}^{2} .
\end{aligned}
$$

De donde para una constante $C$ y procediendo de manera análoga a la prueba del Teorema 3.3 de [9] obtenemos

$$
\frac{1}{|\lambda|^{2}}\|U\|_{H} \leq C\|F\|_{H}
$$

Paso 3: Conclusión. Usando el Teorema 1.4 dado en [2] nuestro resultado de decaimiento polinomial es obtenido como uno deseaba.

\section{Falta de estabilidad exponencial}

Probamos ahora la falta de estabilidad exponencial delsistema de Timoshenko (8)-(13). En esta sección primero mostraremos algunos resultados preliminares para el mejor entendimiento a la hora de la prueba del principal resultado de esta sección que es el Teorema 4.1. Más precisamente, tenemos como objetivo demostrar el siguiente resultado.

Teorema 4.1 El sistema de Timoshenko (8)-(13) con carga puntual no es exponencialmente estable.

Antes vamos a presentar algunas notaciones y resultados preliminares. 
Denotamos por $\{S(t)\}$ el semigrupo definido por el sistema (8)-(13). Note primero que la solución $\varphi(L)$ y $\psi(L)$, de las condiciones de contorno (11) y (12) puden ser escritas como

$$
\varphi_{t}(L, t)=e^{-\frac{k_{0}}{m} t} \varphi_{1}(L)+\frac{1}{m} \int_{0}^{t} e^{-\frac{k_{0}}{m}(t-s)} S(L, s) d s
$$

y

$$
\psi_{t}(L, t)=e^{-\frac{k_{1}}{I_{m}} t} \psi_{1}(L)+\frac{1}{I_{m}} \int_{0}^{t} e^{-\frac{k_{1}}{I_{m}}(t-s)} M(L, s) d s .
$$

donde $S(L, t)=\kappa(L)\left(\varphi_{x}(L, t)+\psi(L, t)\right)$ y $M(L, t)=b(L) \psi_{x}(L, t)$ donde $(x, t) \in(0, L) \times$ $(0, T)$.

Vamos usar las siguientes notaciones

$$
\begin{aligned}
& D_{1}(t)=e^{-\frac{k_{0}}{m} t} \varphi_{1}(L) \\
& K_{1}(t)=\frac{1}{m} \int_{0}^{t} e^{-\frac{k_{0}}{m}(t-s)}\left[\varphi_{x}(L, s)+\psi(L, s)\right] d s \\
& D_{2}(t)=e^{-\frac{k_{1}}{I_{m}} t} \psi_{1}(L), \\
& K_{2}(t)=\frac{1}{I_{m}} \int_{0}^{t} e^{-\frac{k_{1}}{I_{m}}(t-s)} \psi_{x}(L, s) d s
\end{aligned}
$$

Presentaremos ahora un sistema de Timoshenko conservativo

$$
\begin{aligned}
\rho_{1}(x) \widetilde{\varphi}_{t t}-\widetilde{S}_{x} & =0, \\
\rho_{2}(x) \widetilde{\psi}_{t t}-\widetilde{M}_{x}+\widetilde{S} & =0,
\end{aligned}
$$

donde $\widetilde{S}=k(x)\left(\widetilde{\varphi}_{x}+\widetilde{\psi}\right)$ y $\widetilde{M}=b(x) \widetilde{\psi}_{x}$

Las condiciones iniciales son dadas por

$$
\widetilde{\varphi}(x, 0)=\varphi_{0}(x), \widetilde{\varphi}_{t}(x, 0)=\varphi_{1}(x), \widetilde{\psi}(x, 0)=\psi_{0}(x), \widetilde{\psi}_{t}(x, 0)=\psi_{1}(x)
$$

y para el sistema vamos a considerar condiciones de contorno dadas por

$$
\begin{aligned}
\widetilde{\varphi}(0, t) & =\widetilde{\psi}(0, t)=0, \\
\widetilde{S}(L, t)+m \widetilde{\varphi}_{t t}(L, t) & =\widetilde{M}(L, t)+I_{m} \widetilde{\psi}_{t t}(L, t)=0 .
\end{aligned}
$$

Denotando por $\widetilde{E}(t)$ la energía asociada del sistema (50)-(54), usando la norma del sistema (50)-(51) para obtenerla

$$
\widetilde{E}(t)=\frac{1}{2} \int_{0}^{L}\left[\rho_{1}\left|\widetilde{\varphi}_{t}\right|^{2}+\rho_{2}\left|\widetilde{\psi}_{t}\right|^{2}+b|\widetilde{\Psi}|^{2}+k\left|\widetilde{\varphi}_{x}+\widetilde{\psi}\right|^{2}\right] d x+\frac{m}{2}\left|\widetilde{\varphi}_{t}(L, t)\right|^{2}+\frac{I_{m}}{2}\left|\widetilde{\psi}_{t}(L, t)\right|^{2} .
$$

Multiplicando (50) por $\widetilde{\varphi}$ y (51) por $\widetilde{\psi}$ e integrando sobre el intervalo $(0, L)$ obtenemos

$$
\frac{d}{d t} \widetilde{E}(t)=0 \Rightarrow \widetilde{E}(t)=\widetilde{E}(0)
$$

Denotamos por $\left\{S_{0}(t)\right\}$ el semigrupo definido por el sistema (50)-(52) generado por $A_{1}$ con $k_{1}=k_{2}=0$. Note que $\left\|S_{0}(t) U_{0}\right\|=\left\|U_{0}\right\|$. Similarmente, las condiciones de contorno $(53)$ pueden ser escritas como sigue

$$
\widetilde{\varphi}(0, t)=\widetilde{\psi}(0, t)=0
$$


Resolviendo (53) y (54) obtenemos

$$
\begin{aligned}
& \widetilde{\varphi}_{t}(L, t)=\widetilde{\varphi}_{t}(L, 0)+\frac{1}{m} \int_{0}^{t} \widetilde{S}(L, s) d s \\
& \widetilde{\psi}_{t}(L, t)=\widetilde{\psi}_{t}(L, 0)+\frac{1}{m} \int_{0}^{t} \widetilde{M}(L, s) d s
\end{aligned}
$$

Denotamos por

$$
\begin{aligned}
D_{3} & =\varphi_{1}(L), \\
K_{3}(t) & =\frac{1}{m} \int_{0}^{t}\left[\widetilde{\varphi}_{x}(L, s)+\widetilde{\psi}(L, s)\right] d s, \\
D_{4} & =\psi_{1}(L), \\
K_{4}(t) & =\frac{1}{m} \int_{0}^{t} \widetilde{\psi}_{x}(L, s) d s .
\end{aligned}
$$

Usando las notaciones de arriba, tenemos que $U=\varphi-\widetilde{\varphi}$ e $V=\psi-\widetilde{\psi}$ satisface

$$
\begin{aligned}
\rho_{1}(x) U_{t t}-\left[\kappa(x)\left(U_{x}+V\right)\right]_{x} & =0, \\
\rho_{2}(x) V_{t t}-\left[b(x) V_{x}\right]_{x}+k(x)\left(U_{x}+V\right) & =0,
\end{aligned}
$$

con condición inicial

$$
U(x, 0)=U_{t}(x, 0)=V(x, 0)=V_{t}(x, 0)=0
$$

y condiciones de contorno

$$
U(0, t)=V(0, t)=0, U_{t}(L, t)=G_{1}(t), V_{t}(L, t)=G_{2}(t)
$$

donde

$$
\begin{aligned}
& G_{1}(t)=D_{1}(t)-D_{3}+K_{1}(t)-K_{4}(t), \\
& G_{2}(t)=D_{2}(t)-D_{4}+K_{2}(t)-K_{3}(t) .
\end{aligned}
$$

Vamos también a usar las siguientes notaciones

$$
\begin{aligned}
\mathcal{E}(Y, Z, t) & =\int_{0}^{t}\left[\rho_{1}\left|Y_{t}\right|^{2}+\rho_{2}\left|Z_{t}\right|^{2}+b\left|Z_{x}\right|^{2}+\kappa\left|Y_{x}+Z\right|^{2}\right] d x, \\
I_{Y, Z}(t) & =\left|Y_{t}(L, t)\right|^{2}+\left|Y_{x}(L, t)+Z(L, t)\right|^{2} \\
J_{Z}(t) & =\left|Z_{t}(L, t)\right|^{2}+\left|Z_{x}(L, t)\right|^{2}
\end{aligned}
$$

Como en el Lema 3.2 tenemos una estimativa para el siguiente sistema de evolución

Lema 4.2 Suponemos que existe una solución débil para (68)-(69).

$$
\begin{aligned}
\rho_{1} Y_{t t}-\kappa\left(Y_{x}+Z\right)_{x} & =0 \\
\rho_{2} Z_{t t}-b Z_{x x}+\kappa\left(Y_{x}+Z\right) & =0
\end{aligned}
$$

con energía finita $\mathcal{E}(Y, Z, t)$ en $L^{1}(0, t)$. Entonces existe una constante positiva tal que

$$
\int_{0}^{T}\left[I_{Y, Z}(t)+J_{Y}(t)\right] d t \leq C \int_{0}^{T} \mathcal{E}(s) d s+C[\mathcal{E}(T)+\mathcal{E}(0)] .
$$


Además de eso, para $T$ suficientemente grande, tenemos que existe una constante positiva tal que la siguiente desigualdad inversa es satisfecha

$$
\int_{0}^{T} \mathcal{E}(s) d s \leq C \int_{0}^{T}\left[I_{Y, Z}(t)+J_{Z}(t)\right] d t+C[\mathcal{E}(T)+\mathcal{E}(0)] .
$$

Demostración: Multiplicando la ecuación (62) por $q\left(\varphi_{x}+\psi\right)$ y la ecuación (63) por $q \psi_{x}$. Prosiguiendo de forma análoga a la prueba del Lema 3.2 obtenemos nuestro resultado.

Lema 4.3 Considere los siguientes operadores

$$
\begin{aligned}
D_{i}: H & \rightarrow L^{2}(0, T) \\
U_{0} & \mapsto D_{i}(t)
\end{aligned}
$$

$y$

$$
\begin{aligned}
K_{i}: H & \rightarrow L^{2}(0, T) \\
U_{0} & \mapsto K_{i}(t)
\end{aligned}
$$

donde $D_{i}(t), K_{i}(t), i=1,2,3,4$, son definidos en (46), (49), (58), (61) con $(U=\varphi-\widetilde{\varphi}, V=$ $\psi-\widetilde{\psi})$ solución del sistema $(62)-(63)$ y $(\widetilde{\varphi}, \widetilde{\psi})$ solución del sistema (50)-(54). Entonces estos operadores son compactos.

Demostración: Combinando la identidad de energía con el Lema 4.2, obtenemos que las siguientes aplicaciones

$$
\begin{aligned}
& \mathbb{R}^{+} \ni t \mapsto S(L, t), \\
& \mathbb{R}^{+} \ni t \mapsto M(L, t),
\end{aligned}
$$

son limitadas en $L^{2}(0, T)$. Esto implica que la función $\mathbb{R}^{+} \ni t \mapsto K_{i}\left(U_{0}, t\right)$ lleva conjuntos limitados de $H$ en conjuntos limitados de $H^{1}(0, T)$. Por tanto, por uno de los Teoremas de imersión dado en el Teorema 2.5.1 dado en [1] la aplicación $\mathbb{R}^{+} \ni t \mapsto K_{i}\left(U_{0}, t\right)$ es compacta de $H$ en $L^{2}(0, T)$.

\subsection{Prueba del Teorema 4.1}

Usaremos los siguientes enunciados y resultados para nuestra prueba.

Definición 4.4 El tipo de semigrupo $\{S(t)\}$ es definido por el siguiente número

$$
\omega\left(A_{1}\right)=\lim _{t \rightarrow \infty} \frac{\ln (\|S(t)\|)}{t}=\inf _{t>0} \frac{\ln (\|S(t)\|)}{t} .
$$

Definición 4.5 El tipo esencial del semigrupo $\{S(t)\}$ es definido por

$$
\omega_{\text {ess }}\left(A_{1}\right)=\inf \left\{\omega \in \mathbb{R} \mid \exists M>0 \text { tal que }\|S\|_{\text {ess }} \leq M e^{\omega t}, \forall t \geq 0\right\}
$$

donde $\|S\|_{\text {ess }}=\inf \{\|S-K\|, K$ compacto $\}$.

Notemos que $\|S\|_{\text {ess }} \leq\|S\|$ 
Definición 4.6 Sea $\omega_{\sigma}\left(A_{1}\right)$ el límite superior espectral del conjunto resolvente $\sigma\left(A_{1}\right)$.

Observación 4.7 Note que $\omega\left(A_{1}\right)=0$ implica $\|S(t)\|=1$. Por tanto para probar la falta de estabilidade exponencial, es suficiente provar que $\omega\left(A_{1}\right)=0$.

Teorema 4.8 (Weyl) El radio esencial de espectro de un operador $A_{1}$ es invariante por perturbaciones compactas, o sea

$$
r_{\text {ess }}\left(A_{1}\right)=r_{\text {ess }}\left(A_{1}+K\right)
$$

donde $K$, es un operador compacto y $r_{\text {ess }}\left(A_{1}\right)$ es el radio espectral esencial de $A_{1}$ y es dado por

$$
r_{e s s}\left(A_{1}\right)=\operatorname{máx}\left\{|\lambda| ; \lambda \in \sigma_{e s s}\left(A_{1}\right)\right\}=\inf \left\{R>0 ; \lambda \in \sigma\left(A_{1}\right),|\lambda|>R \Rightarrow \lambda \in \sigma_{d}\left(A_{1}\right)\right\}
$$

y $\sigma_{d}\left(A_{1}\right)$ es un conjunto de valores propios isolados de $A_{1}$ con multiplicidad finita

Demonstração: Ver [4]

Note primero, que si $\omega\left(A_{1}\right)=0$, donde $A_{1}$ es el generador infinitesimal del semigrupo $\{S(t)\}$ de clase $C_{0}$, entonces tenemos que $\|S(t)\|=1$. Así para mostrar la falta de estabilidad exponencial es suficiente mostrar que $\omega\left(A_{1}\right)=0$. Para esto vamos a usar la siguiente fórmula

$$
\omega(S)=\operatorname{máx}\left\{\omega_{\sigma}\left(A_{1}\right), \omega_{e s s}(S)\right\} .
$$

Como $\omega_{\sigma}\left(A_{1}\right) \leq 0$ siempre que $A_{1}$ es disipativo. Entonces la demostración del Teorema 4.1 es resumida a mostrar que $\omega_{\text {ess }}(S)=0$.

Para esto vamos a mostrar primero que la diferencia de los semigrupos $S-S_{0}$ es un operador compacto en $H$ y entonces del Teorema de imersión sigue que

$$
\omega_{e s s}(S)=\omega_{e s s}\left(S_{0}\right) \text {. }
$$

Por tanto, el radio espectral esencial de $\{S(t)\}$ y $\left\{S_{0}(t)\right\}$ son iguales. Siendo $\left\{S_{0}(t)\right\}$ unitario, tenemos que el espectro es dado por 0. Concluyendo entonces que $\omega(S)=0$ y así por la siguiente Observación 4.7 sigue que $\{S(t)\}$ no es exponencialmente estable.

Demostración del Teorema 4.1: Vamos a probar que $S-S_{0}: H \rightarrow H$ es un operador compacto. Con efecto, multiplicando la ecuación (62) y (63) por $U_{t}$ e $V_{t}$, respectivamente, obtenemos

$$
\mathcal{E}_{t}(U, V, t)=S(L, t)\left(D_{1}+K_{1}\right)+M(L, t)\left(D_{2}+K_{2}\right) .
$$

Integrando sobre la variable temporal, encontramos

$$
\mathcal{E}(U, V, t)=\int_{0}^{t}\left[S(L, s)\left(G_{1}\right)+M(L, s)\left(G_{2}\right)\right] d s .
$$

Usando la primera desigualdad del Lema 4.2, obtenemos que

$$
\int_{0}^{T}\left[I_{U, V}(t)+J_{U}(t)\right] d t \leq \sum_{i=1}^{4} \int_{0}^{T}\left[\left|D_{i}\right|^{2}+\left|K_{i}\right|^{2}\right] d s .
$$

De donde sigue que

$$
\int_{0}^{T} \mathcal{E}(U, V, s) d s \leq C \sum_{i=1}^{4} \int_{0}^{T}\left[\left|D_{i}\right|^{2}+\left|K_{i}\right|^{2}\right] d s .
$$

Note que, por el Lema $4.3, D_{i}\left(U_{0}\right)$ y $K_{i}\left(U_{0}\right)$ son conjuntos compactos en $L^{2}(0, T)$ si $U_{0}$ es un conjunto limitado. Así para cualquier sucesión de datos iniciales, $\left\{U_{0}^{\mu}\right\}$, limitada en el espacio fase $H$ existe una subsucesión (aún denotada de la misma manera) tal que $\left\{D_{i} U_{0}^{\mu}\right\}$ y $\left\{K_{i} U_{0}^{\mu}\right\}$ convergen fuerte en $L^{2}(0, T)$. Por la linearidad tenemos que $\left\{U^{\mu}\right\},\left\{V^{\mu}\right\}$ también convergen fuerte en $H$ y $\left\{\left(S-S_{0}\right)\left(U_{0}^{\mu}\right)\right\}$ converge fuerte. Por tanto $S-S_{0}$ es un operador compacto. Con esto se concluye la demostración. 


\section{Abordaje Computacional y Discretización del Sistema}

Observación 5.1 Para nuestro análisis, los coeficientes serán constantes.

\subsection{Discretización del Sistema}

Hacemos la discretización del sistema usando diferencias finitas para las variables temporal y espacial, para las derivadas de orden 2 usamos diferencia centrada y para las derivadas de orden 1 usamos o método explícito.

La discretización del tiempo es dada por

$$
t_{0}=0<t_{1}=\Delta t<\ldots<t_{n}<\ldots<t_{N}=N \Delta t<t_{N+1}=T
$$

donde $t_{n}=n \Delta t$ para $n=0,1,2, \ldots N+1 \mathrm{y}$ hacemos

$$
h=\Delta x=\frac{L}{i+1}, \Delta t=\frac{T}{N+1}, L, T \in \mathbb{N}
$$

$\operatorname{con} x_{i}=i \Delta x, i=0,1,2, \ldots, I+1$ e $t_{n}=n \Delta t$.

Consideremos también los siguientes operadores de diferencias finitas en espacio y en el tiempo:

Esquema Progresivo Implícito (primer orden):

$$
\partial_{x} u_{j}^{n}=\frac{u_{j+1}^{n}-u_{j}^{n}}{\Delta x}, \quad \partial_{t} u_{j}^{n}=\frac{u_{j}^{n+1}-u_{j}^{n}}{\Delta t}
$$

Esquema Atrasado Explícito (primer orden):

$$
\bar{\partial}_{x} u_{j}^{n}=\frac{u_{j}^{n}-u_{j-1}^{n}}{\Delta x}, \quad \bar{\partial}_{t} u_{j}^{n}=\frac{u_{j}^{n}-u_{j}^{n-1}}{\Delta t}
$$

Diferencia Central (segundo orden):

$$
\frac{\partial_{x}+\bar{\partial}_{x}}{2} u_{j}^{n}=\frac{u_{j+1}^{n}-u_{j-1}^{n}}{2 \Delta x}, \quad \frac{\partial_{t}+\bar{\partial}_{t}}{2} u_{j}^{n}=\frac{u_{j}^{n+1}-u_{j}^{n-1}}{2 \Delta t}
$$

Esquema de Diferencias Centrada (segundo orden)

$$
\bar{\partial}_{x} \partial_{x} u_{j}^{n}=\frac{u_{j+1}^{n}-2 u_{j}^{n}+u_{j-1}^{n}}{\Delta x^{2}}, \quad \bar{\partial}_{t} \partial_{t} u_{j}^{n}=\frac{u_{j}^{n+1}-2 u_{j}^{n}+u_{j}^{n-1}}{\Delta t^{2}},
$$

En todos los casos $u_{j}^{n}$ corresponde a la solución numérica en los puntos nodales $\left(x_{j}, t_{n}\right)$ de la discretización, y tales operadores son construidos con base en la aplicación de la serie de Taylor para $u\left(x_{j}, t_{n}\right)$.

\subsection{Convergencia del Esquema Numérico}

Definición 5.2 Decimos que un esquema numérico en diferencias finitas expresado por

$$
F_{(\Delta x, \Delta t)} u(\Delta x, \Delta t)=0
$$

y que aproxima una ecuación diferencial parcial $F u(x, t)=0$, es convergente en cada punto $\left(x_{j}, t_{n}\right)$ si para $\left(x_{j}, t_{n}\right) \longrightarrow(x, t)$ tenemos que $u_{j}^{n} \rightarrow u$ cuando $\Delta x, \Delta t \rightarrow 0$. Formalmente

$$
\lim _{\Delta x \rightarrow 0} \operatorname{máx}_{j=0,1,2, \ldots} \lim _{\Delta t \rightarrow 0} \operatorname{máx}_{n=0,1,2, \ldots}\left|u\left(x_{j}, t_{n}\right)-u_{j}^{n}\right|=0
$$


Así nuestro sistema (8)-(13) queda expresado de la siguiente manera

$$
\begin{aligned}
& \rho_{1}\left(\frac{\varphi_{i}^{n+1}-2 \varphi_{i}^{n}+\varphi_{i}^{n-1}}{\Delta t^{2}}\right)-\kappa\left(\frac{\varphi_{i+1}^{n}-2 \varphi_{i}^{n}+\varphi_{i-1}^{n}}{h_{x}^{2}}+\frac{\psi_{i}^{n}-\psi_{i-1}^{n}}{h_{x}}\right)=0, \\
& \rho_{2}\left(\frac{\psi_{i}^{n+1}-2 \psi_{i}^{n}+\psi_{i}^{n-1}}{\Delta t^{2}}\right)-b\left(\frac{\psi_{i+1}^{n}-2 \psi_{i}^{n}+\psi_{i-1}^{n}}{h_{x}^{2}}\right)+\kappa\left(\frac{\varphi_{i}^{n}-\varphi_{i-1}^{n}}{h_{x}}\right)-\kappa \psi_{i}^{n}=0 .
\end{aligned}
$$

De (70) tenemos

$$
\frac{\rho_{1}}{\Delta t^{2}} \varphi_{i}^{n+1}-\frac{2 \rho_{1}}{\Delta t^{2}} \varphi_{i}^{n}+\frac{\rho_{1}}{\Delta t^{2}} \varphi_{i}^{n-1}=\frac{\kappa}{h_{x}^{2}} \varphi_{i+1}^{n}-\frac{2 \kappa}{h_{x}^{2}} \varphi_{i}^{n}+\frac{\kappa}{h_{x}^{2}} \varphi_{i-1}^{n}+\frac{\kappa}{h_{x}} \psi_{i}^{n}-\frac{\kappa}{h_{x}} \psi_{i-1}^{n} .
$$

De (71) tenemos

$$
\frac{\rho_{2}}{\Delta t^{2}} \psi_{i}^{n+1}-\frac{2 \rho_{2}}{\Delta t^{2}} \psi_{i}^{n}+\frac{\rho_{2}}{\Delta t^{2}} \psi_{i}^{n-1}=\frac{b}{h_{x}^{2}} \psi_{i+1}^{n}-\frac{2 b}{h_{x}^{2}} \psi_{i}^{n}+\frac{b}{h_{x}^{2}} \psi_{i-1}^{n}-\frac{\kappa}{h_{x}} \varphi_{i-1}^{n}+\frac{\kappa}{h_{x}} \varphi_{i-1}^{n}-\kappa \psi_{i}^{n} .
$$

La condición de contorno en $x=L$ es escrita como

$$
\begin{aligned}
m\left(\frac{\varphi_{i}^{n+1}-2 \varphi_{i}^{n}+\varphi_{i}^{n-1}}{\Delta t^{2}}\right)+k_{0}\left(\frac{\varphi_{i}^{n}-\varphi_{i}^{n-1}}{\Delta t}\right)+\kappa\left(\frac{\varphi_{i}^{n}-\varphi_{i-1}^{n}}{h_{x}}\right)+\kappa \psi_{i}^{n} & =0 \\
I_{m}\left(\frac{\psi_{i}^{n+1}-2 \psi_{i}^{n}+\psi_{i}^{n-1}}{\Delta t^{2}}\right)+k_{1}\left(\frac{\psi_{i}^{n}-\psi_{i}^{n-1}}{\Delta t}\right)+b\left(\frac{\psi_{i}^{n}-\psi_{i-1}^{n}}{h_{x}}\right) & =0
\end{aligned}
$$

De (72) tenemos

$$
\frac{m}{\Delta t^{2}} \varphi_{i}^{n+1}-\frac{2 m}{\Delta t^{2}} \varphi_{i}^{n}+\frac{m}{\Delta t^{2}} \varphi_{i}^{n-1}+\frac{k_{0}}{\Delta t} \varphi_{i}^{n}-\frac{k_{0}}{\Delta t} \varphi_{i}^{n-1}+\frac{\kappa}{h_{x}} \varphi_{i}^{n}-\frac{\kappa}{h_{x}} \varphi_{i-1}^{n}+\kappa \psi_{i}^{n}=0
$$

De (73) tenemos

$$
\frac{I_{m}}{\Delta t^{2}} \psi_{i}^{n+1}-\frac{2 I_{m}}{\Delta t^{2}} \psi_{i}^{n}+\frac{I_{m}}{\Delta t^{2}} \psi_{i}^{n-1}+\frac{k_{1}}{\Delta t} \psi_{i}^{n}-\frac{k_{1}}{\Delta t} \psi_{i}^{n-1}+\frac{b}{h_{x}} \psi_{i}^{n}-\frac{b}{h_{x}} \psi_{i-1}^{n}=0
$$

\subsection{Consistencia y Estabilidad}

En particular, el concepto de consistencia nos dice que la solución exacta de un problema de valor inicial y de contorno satisface las ecuaciones de diferencias de los métodos numéricos con un margen de error dado, que se obtiene de la aproximación via serie de Taylor.

Definición 5.3 Para $F u(x, t)=0$ y $F_{(\Delta x, \Delta t)} u(\Delta x, \Delta t)=0$, decimos que el esquema numérico es consistente si para cualquier función $u=u(x, t)$ suficientemente regular se tiene que:

$$
F_{(\Delta x, \Delta t)} u(\Delta x, \Delta t) \rightarrow 0 \text { cuando }(\Delta x, \Delta t) \rightarrow 0
$$

Definición 5.4 Un método de diferencias finitas es estable si existe una constante $M>0$, tal que, $\left|e_{j}^{n}\right|<M$ para todo $j, n$.

Teorema 5.5 (Convergencia Numérica) El esquema numérico explícito espacio-tiempo aplicado al sistema, es convergente sí, y solamente sí,

$$
\Delta t \leq \frac{\Delta x}{c}
$$

donde $c=\sqrt{E / p}$.

Demostración: Ver $[15,11]$.

Observación 5.6 (Propiedades de la Energía Discreta del Método Explícito) Para la discretización de la energía usaremos también diferencias finitas en la variable espacial $x$. 


\subsection{Resultados Computacionales}

Ahora exhibimos los resultados de la simulación numérica que efectuamos del método explícito en diferencias finitas, principalmente lo que nos muestra es la falta de decaimiento exponencial de la energía. La principal cuestión es que tratándose de un modelo de dimensión finita, se debe determinar los parámetros adecuados para asegurar buenos resultados.

Usamos los siguientes parámetros dados en [1]. Por tanto consideramos el caso con una viga de longitud $L=1$, con las siguientes constantes físicas $E=1 \times 10^{4} \mathrm{~N} / \mathrm{m}^{2}, G=4,12 \times 10^{4} \mathrm{~N} / \mathrm{m}^{2}, \rho=$ $1500 \mathrm{~kg} / \mathrm{m}^{3}$ y $K=2 / 6$.

Con las siguientes condiciones iniciales:

$$
\varphi(x, 0)=\psi(x, 0)=8 \operatorname{sen}\left(4 \pi \frac{x}{L}\right), \varphi_{t}(x, 0)=\psi_{t}(x, 0)=8 \operatorname{sen}\left(8 \pi \frac{x}{L}\right) .
$$

Usamos estas funciones ya que el sistema (8)-(13) tiene condiciones de Dirichlet por la izquierda $x=0$ y la carga puntual funciona como si también tuviera la misma condición a la derecha $x=L$. Usamos una malla adecuada en cada caso usando la condición (74) y la evolución del tiempo hasta $t=10$.

Se obtuvieron los siguientes resultados.

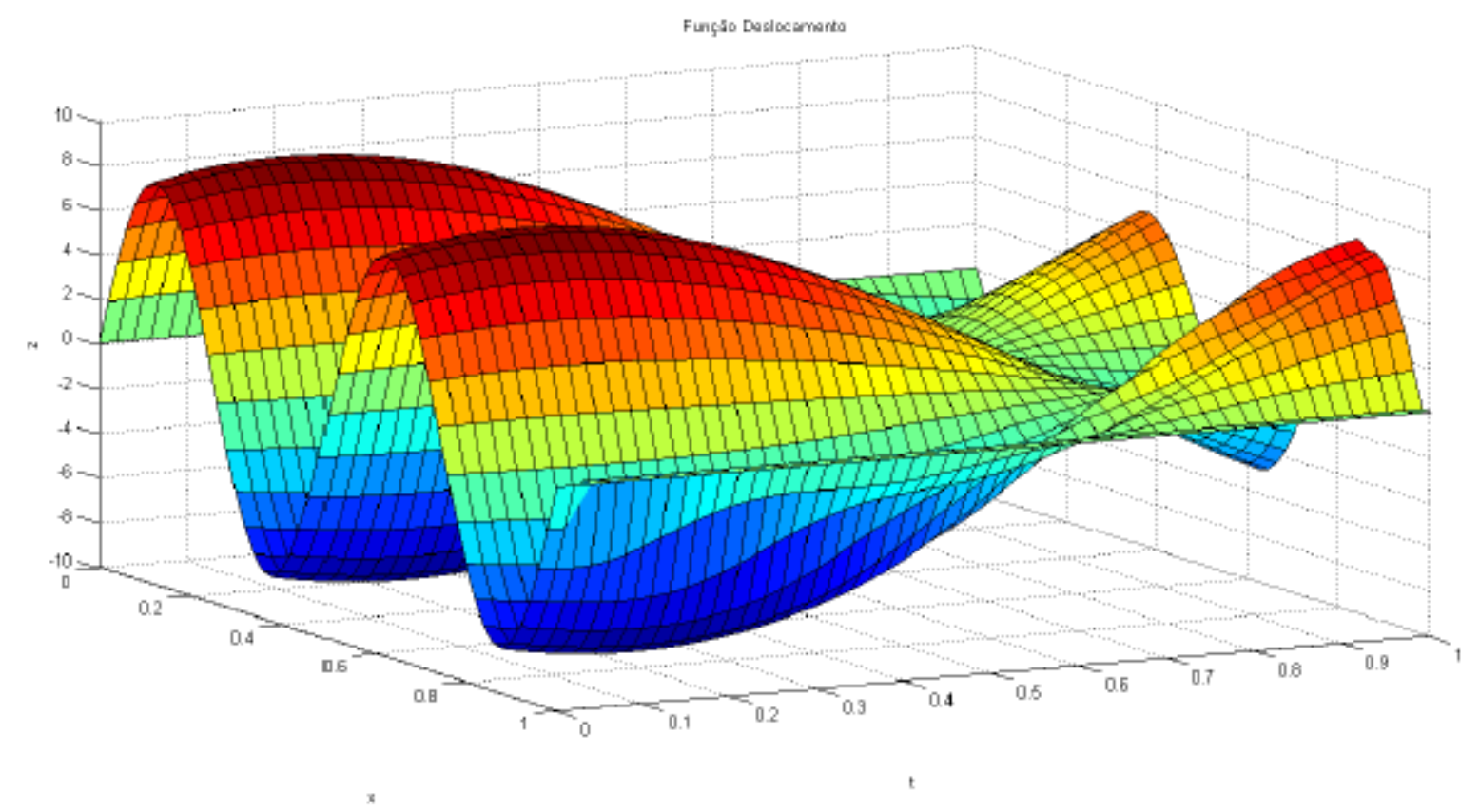

Figura 2: Caso 1 Función Desplazamiento para $t=1$. 


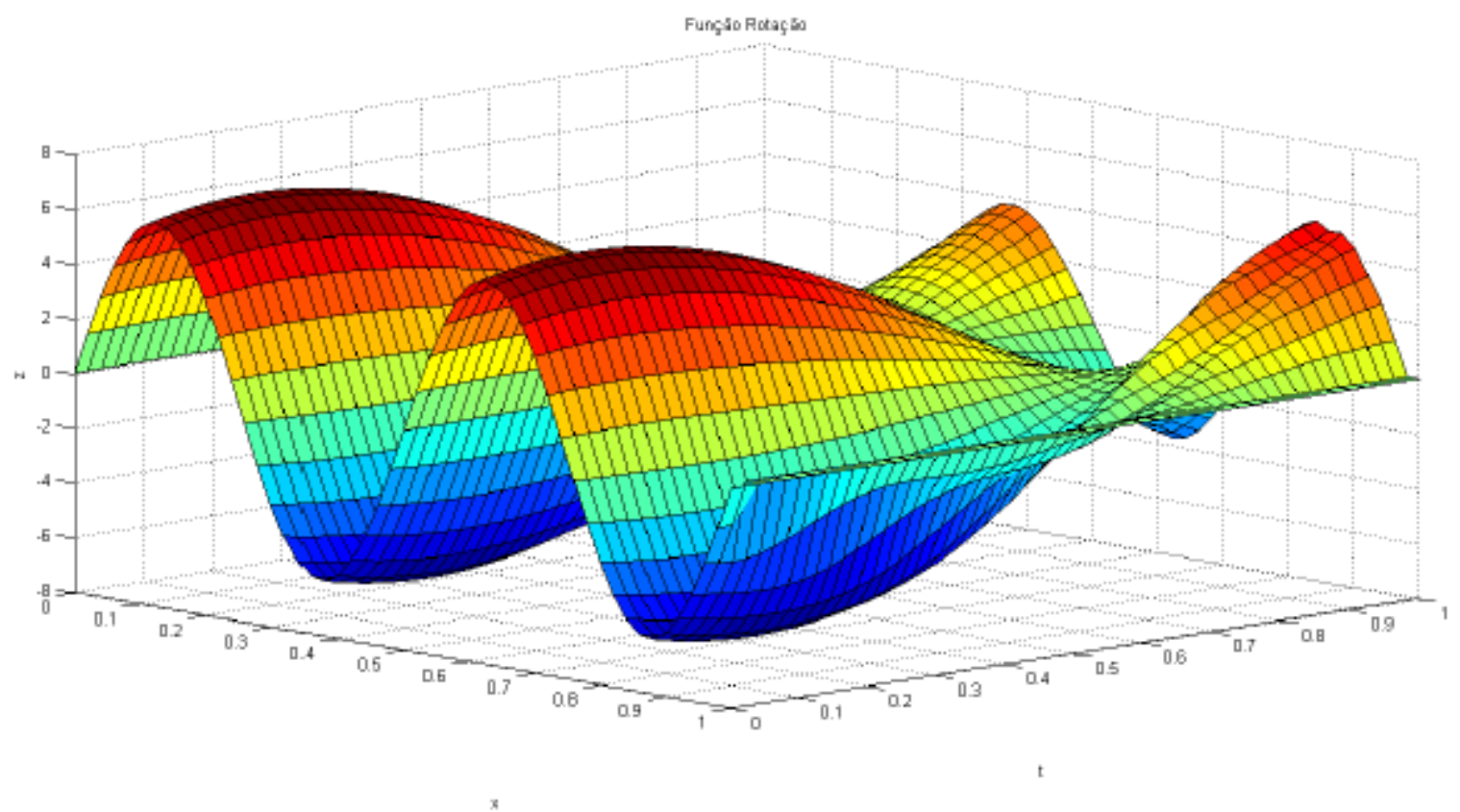

Figura 3: Caso 1 Función de Rotación para $t=1$.

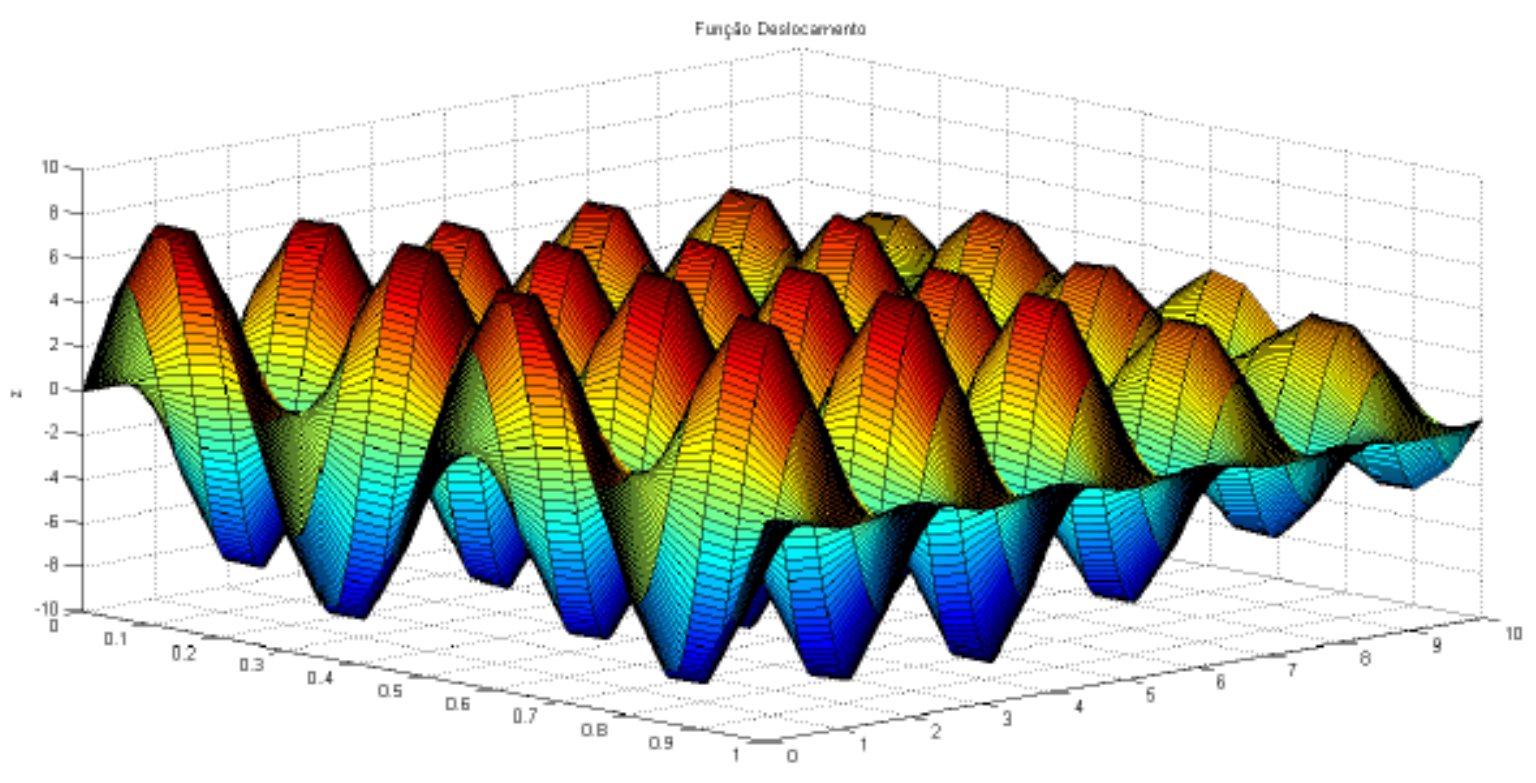

Figura 4: Función Desplazamiento $\varphi$ para $t=10$. 


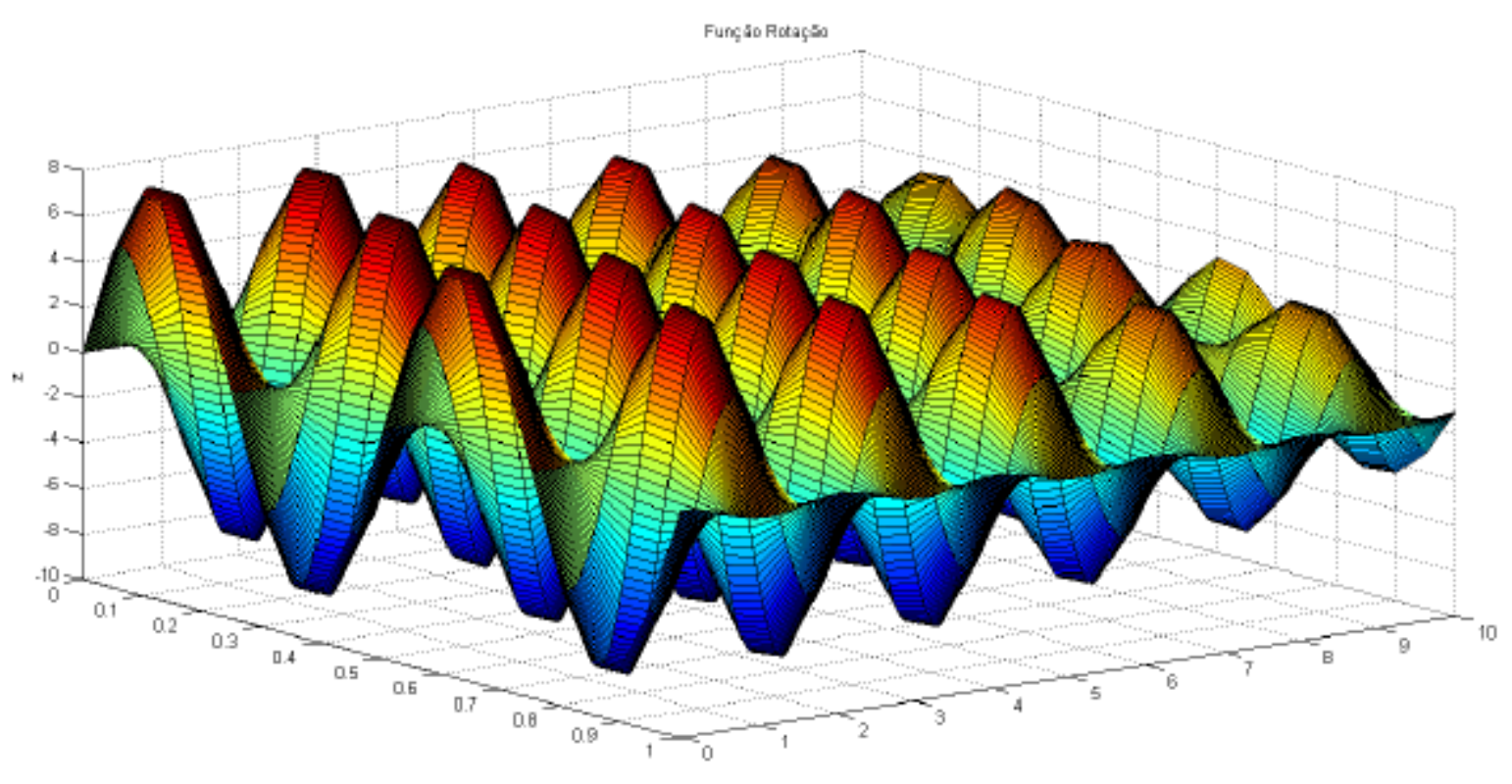

Figura 5: Función Rotación $\psi$ para $t=10$.

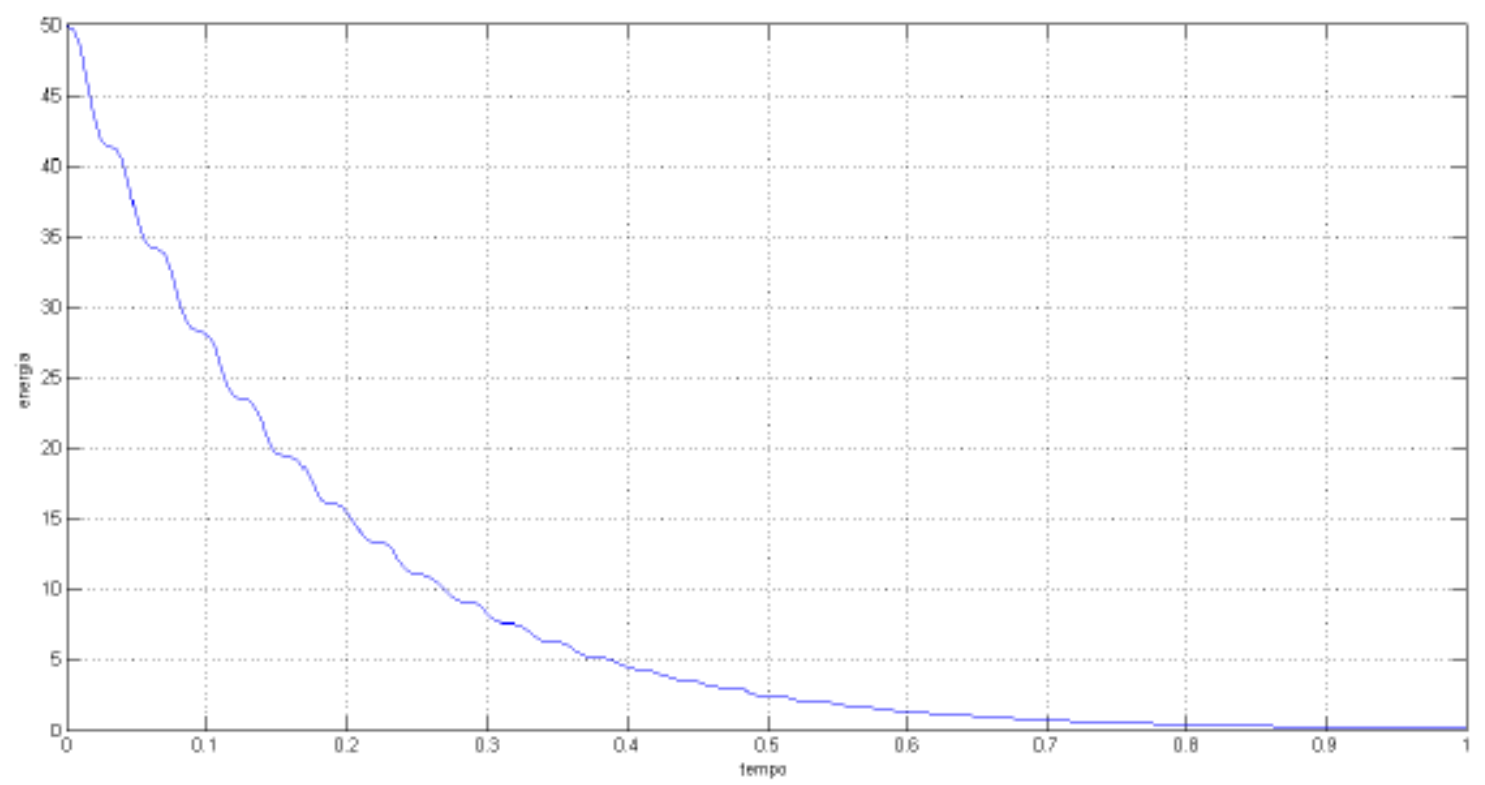

Figura 6: Decaimiento de la Energía 


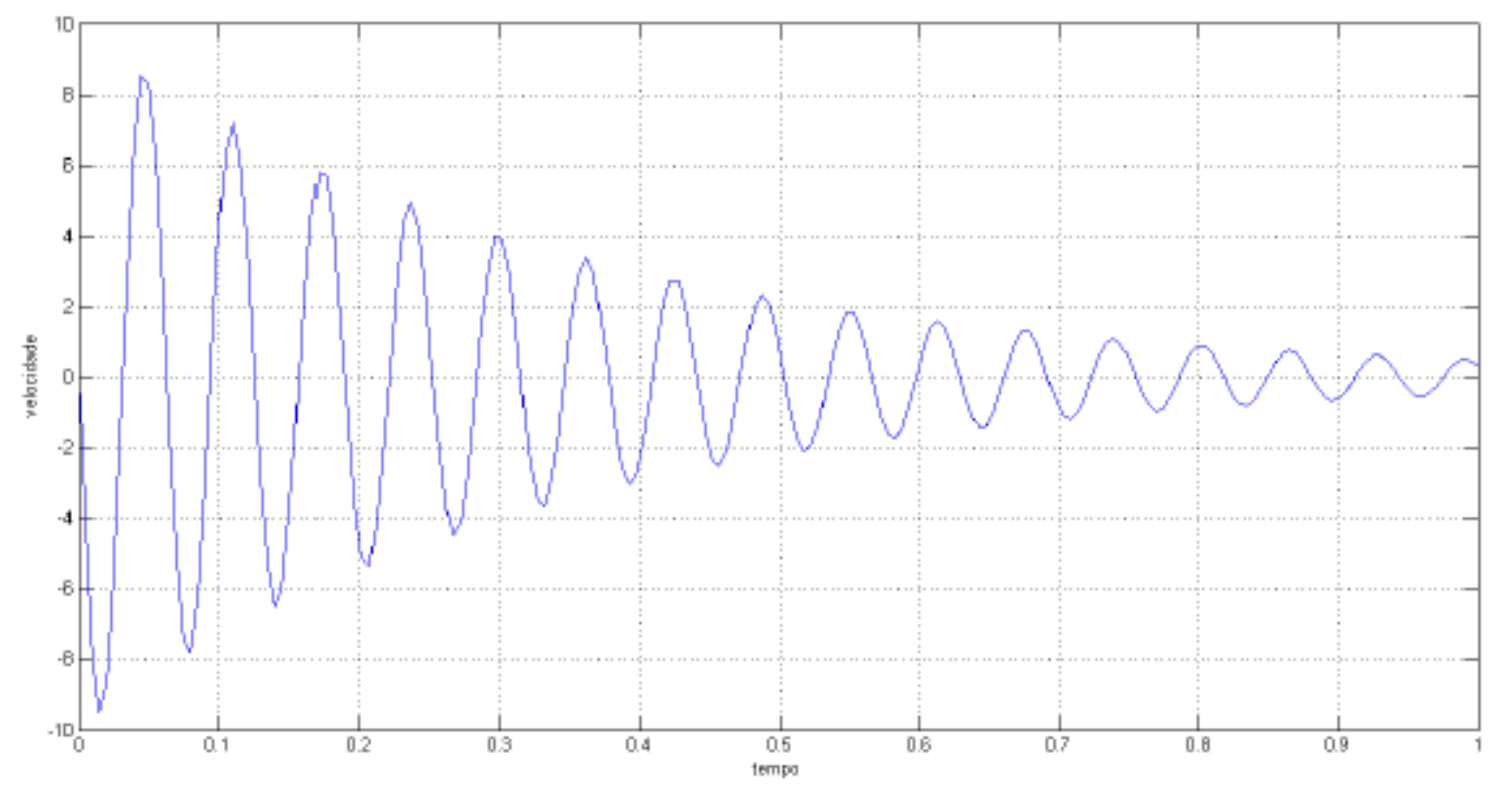

Figura 7: Velocidad de las vibraciones

De las figuras observamos lo siguiente:

- En las Figuras 2 y 3 , observamos el comportamiento en el tiempo $t=1$ de las funciones Desplazamiento $\varphi$ y Rotación $\psi$, donde se puede ver un comportamiento similar debido a que usamos las mismas condiciones iniciales para ambas funciones.

- En las Figuras 4 y 5 , observamos el comportamiento en el tiempo $t=10$ de las funciones Desplazamiento $\varphi$ y Rotación $\psi$, donde se puede ver un comportamiento similar debido a que ambas poseen mecanismo disipativo dado en la frontera $x=L$; esto a diferencia de otros modelos en donde por ejemplo el sistema tiene disipación parcial, como es el caso visto en el Capítulo 4 de [1], en estos casos el comportamiento de las funciones $\varphi$ y $\psi$ y sus tazas de decaimiento serían diferentes.

- En la Figura 6 observamos el decaimiento de la energía, su comportamiento no es exponencial debido a la taza de decaimiento polinomial de nuestro sistema. En la Figura 7, observamos la evolución de las ondas, estas no decaen rápidamente debido a que el sistema (8)-(13) tiene decaimiento polinomial.

\section{Conclusión}

- En este trabajo el resultado principal es la prueba de la ausencia de estabilidad exponencial del sistema presentado. A su vez se muestra que el sistema tiene estabilidad polinomial, ambos casos son demostrados durante el trabajo.

- En lo que respecta a la implementación computacional obervamos las vibraciones, la velocidad de las ondas que suceden en la viga con el pasar del tiempo y la falta de estabilidad exponencial en su decaimiento. 
- También observamos en la gráfica de la energía la taza de decaimento polinomial del problema. Para otros trabajos se piensa hacer análisis e implementaciones con otros tipos de disipación.

\section{Referencias bibliográficas}

[1] Acasiete, F. (2016). Modelagem computacional da viga de Timoshenko submetida a cargas pontuais (tese de mestrado). Laboratório Nacional de Computação Científica, Rio de Janeiro, Brasil.

[2] Borichev, A., y Tomilov, Y. (2009). Optimal polynomial decay of functions and operator semigroups. Mathematische Annalen, 347(2), 455-478.

[3] Chipana, E. (2010). Estabilização Assintótica e Controle no Problema de Conexões em Série de Vigas (tese de mestrado). Laboratório Nacional de Computação Científica, Rio de Janeiro, Brasil.

[4] Gohberg, I. C. y Krein, M. G. (1969). Introduction to the theory of linear non self adjont operators in Hilbert Space. Providence, Rhode Island: American Mathematical Society.

[5] Kafini, M. (2011). General energy decay in a Timoshenko-type system of thermo-elasticity of type III with a viscoelastic damping. Journal of Mathematical Analysis and Applications, $375(2), 523-537$

[6] Kim, J. U. y Renardy, Y. (1987). Boundary control of the timoshenko beam. Society for Industrial and Applied Mathematics, 25(6), 1417-1429.

[7] Messaoudi, S. A. y Said-Houari, B. (2008). Energy decay in a Timoshenko-type system of thermo-elasticity of type III. Journal of Mathematical Analysis and Applications, 348(1), 298-307.

[8] Messaoudi,S. A. y Mustafa, M.I. (2008). On the internal and boundary stabilization of Timoshenko beams. Nonlinear Differential Equations and Applications NoDEA, 15(6), 655671.

[9] Muñoz Rivera, J. E. y Avila, A. I. (2013). Rates of decay to non homogeneous timoshenko model with tip body. Journal of Differential Equations, 258,(10), 3468-3490.

[10] Muñoz Rivera, J. E. (2009). Estabilização de Semigrupos e Aplicações. Rio de Janeiro, Brasil: Laboratório Nacional de Computação Científica.

[11] Negreanu, M. y Zuazua, E. (2003). Uniform boundary controllability of a discrete 1-D wave equation. System and Control Letters, 48(3-4), 261-280.

[12] Pazy, A. (1983) Semigroups of Linear Operators and applications to partial Differential Equations. New York, United States of America: Springer.

[13] Raposo, C. A., Ferreira, J., Santos, M. L. y Castro, N. N. O. (2005). Exponential stability for the timoshenko system with two weak dampings. Applied Mathematics Letters, 18(5), 535-541

[14] Soufyane, A. y Wehbe, A. (2003). Uniform stabilization for the timoshenko beam by a locally distributed damping. Electronic Journal of Differential Equations, 2003(29), 1-14 
[15] Strauss, W. y Vasquez, L. (1978). Numerical solution of a nonlinear klein-gordon equation. Journal of Computational Physics, 28(2), 271-278.

[16] Timoshenko, S. P. (1921). LXVI. On the correction for shear of the differential equation for transverse vibrations of prismatic bars. The London, Edinburgh, and Dublin Philosophical Magazine and Journal of Science, 41(245), 744-746. doi:10.1080/14786442108636264 